\title{
Optimization of Drug Prescription and Medication Management in Older Adults with Cardiovascular Disease
}

Nanette K. Wenger ${ }^{1}$, Caroline Lloyd Doherty ${ }^{2}$, Jerry H. Gurwitz ${ }^{3}$, Glenn A. Hirsch ${ }^{4}$, Holly M. Holmes ${ }^{5}$, Matthew S. Maurer ${ }^{6}$, Michael D. Murray ${ }^{7}$

${ }^{1}$ Emory University School of Medicine, 49 Jesse Hill Jr. Drive, SE, Atlanta, GA 30303, USA

${ }^{2}$ University of Pennsylvania School of Nursing, Philadelphia, PA, USA

${ }^{3}$ University of Massachusetts School of Medicine, Worcester, MA, USA

${ }^{4}$ University of Louisville School of Medicine, Louisville, KY, USA

${ }^{5}$ McGovern Medical School, University of Texas, Houston, TX, USA

${ }^{6}$ Columbia University Medical Center, New York, NY, USA

${ }^{7}$ Purdue University College of Pharmacy, Indianapolis, IN, USA

Corresponding Author:

Nanette K. Wenger

nwenger@emory.edu

This is the author's manuscript of the article published in final edited form as:

Wenger, N. K., Doherty, C. L., Gurwitz, J. H., Hirsch, G. A., Holmes, H. M., Maurer, M. S., \& Murray, M. D. (2017). Optimization of Drug Prescription and Medication Management in Older Adults with Cardiovascular Disease. Drugs \& Aging, 34(11), 803-810. https://doi.org/10.1007/s40266-017-0494-2 


\section{Key Points}

Most elderly patients with cardiovascular disease have three or more other chronic conditions and thus are prescribed multiple medications.

Polypharmacy at an elderly age predisposes the patient to adverse drug events and non-adherence, with resultant escalation of morbidity, mortality, and healthcare costs.

This manuscript highlights optimization of drug prescription, medication management, and the role of patient goals of care in shared decision-making. 


\section{Abstract}

Cardiovascular disease increases incrementally with age and elderly patients concomitantly sustain multimorbidities, with resultant prescription of multiple medications. Despite conforming with diseasespecific cardiovascular clinical practice guidelines, this polypharmacy predisposes many elderly individuals with cardiovascular disease to adverse drug events and non-adherence. Patient-centered care requires that the clinician explore with each patient his or her goals of care and that this shared decision-making constitutes the basis for optimization of medication management. This approach to aligning therapies with patient preferences is likely to promote patient satisfaction, to limit morbidity, and to favorably affect healthcare costs.

\section{Polypharmacy in Older Adults: Scope of the Problem}

The prevalence of cardiovascular disease increases incrementally with age [1]. The majority of patients with ischemic heart disease, stroke, heart failure, and atrial fibrillation have three or more other chronic conditions (see illustrative case in Table 1). Forty-four percent of men and $57 \%$ of women $>65$ years of age take five or more medications each week; in another report, $37 \%$ of men and $36 \%$ of women aged 75-85 years take at least five prescription medications. Twelve percent of both elderly men and women take ten or more medications weekly; this includes prescription medications, over-the-counter preparations, and herbal products [2].

Patients in the Medicare age population (13\% of the population) take a third of all prescribed medications. As well, these people consume $40 \%$ of all over-the-counter medications. Some polypharmacy may result from a prescribing cascade; that is, medications given to treat adverse effects and not subsequently discontinued [3]. Polypharmacy is associated with an increase in healthcare costs, adverse drug events, drug interactions, medication nonadherence, a decrease in functional status, and geriatric syndromes [4]. Deprescribing - a complex issue that likely warrants a separate manuscript-is described in detail elsewhere [28], but importantly impacts the topics to be discussed subsequently: adverse drug events, medication adherence, medication management, and shared decision-making in older adults with cardiovascular disease and multiple chronic conditions.

Deprescribing must be included in the roles of the primary physician, the pharmacist, the cardiologist, and the entire care team. It is ideally addressed at each transition of care and medication reconciliation.

\section{Adverse Drug Events (ADEs) in Older Adults and Their Increased Vulnerability to ADEs}

Adverse drug events (ADEs) account for more than 170,000 emergency room visits for elderly patients each year [5]. There is a sevenfold increased likelihood that these patients will be admitted to hospital as compared with younger patients. Sixty-seven percent of all ADEs are related to warfarin, insulin, oral antiplatelet agents, and oral hypoglycemics [6], with two thirds of these due to unintentional overdose. ADEs are the fourth leading cause of hospital admission for older adults and represent $25 \%$ of their hospital admissions. Altered mental status is a common component of presentation. Not surprisingly, the risk of ADEs increases exponentially as the medication list expands.

Aging changes in body composition, drug metabolism and clearance contribute to altered drug pharmacokinetics. The physiologic changes of aging impact pharmacodynamics (drug-physiologic 
substrate interaction). As well, drug-disease interactions and drug-drug interactions result from polypharmacy. This includes prescription drugs, over-the-counter drugs, herbal medications, and dietary supplements. Owing to the underreporting or disregard of ADEs, the numbers cited may be an underrepresentation (Tables 2, 3, 4).

\section{Medication Non-Adherence: Scopeof the Problem}

Non-adherence has a significant impact on disease control, morbidity, mortality, and healthcare costs. Non-adherence costs the United States healthcare system over US\$100 billion in avoidable costs annually [7]. Because of the importance of non-adherence as a global health issue, the World Health Organization (WHO) released a report, Adherence to Long-Term Therapies: Evidence for Action in 2003. The WHO proposed a five-dimensional model of adherence that incorporates social and economic factors, therapy-related factors, patient factors, condition-related factors, and health system or healthcare team factors as contributors to adherence [9]. Missing from this valuable model are issues predominating in older adults: factors relevant to individuals with a decrease in life expectancy, severity of comorbidities, impairment of function, and patient priority aligned care. Improving adherence would have a far greater impact on health than developing additional therapies, particularly for chronic diseases such as cardiovascular disease and hypertension; nonetheless, in the elderly patient with multimorbidities, adherence issues must be aligned with patient priorities and goals of care. After receiving a prescription, about $30 \%$ of patients are estimated to not fill the prescription (initial or primary nonadherence).

In a study of 32,514 prescriptions written to 15,961 people in Quebec, Canada, 31.3\% did not fill the prescription within 9 months. Of these prescriptions that were written, $86.7 \%$ were for new therapies and $13.3 \%$ were for a change in therapy. The mean age of patients in this study was 61.55 years, and there was a decreased risk of non-adherence with increasing age. The highest incidence of initial nonadherence was for medications for coronary artery disease (CAD), headache, and depression, with $51.3 \%$ of prescriptions for CAD going unfilled [9].

Higher cost, higher numbers of recent hospitalizations, and the severity of comorbid conditions were all associated with higher rates of non-adherence.

Non-adherence is common, particularly for chronic, asymptomatic conditions such as hypertension and hyperlipidemia. Estimates for non-adherence range from 25 to $50 \%$, depending on the condition, treatment, and the method to estimate adherence [10]. Self-reported non-adherence ranges from 33 to $57 \%$ [11]. Cost-related nonadherence (CRN) is a particularly important factor as nonadherence may be related to additional disparities in care by racial/ethnic group and socioeconomic status and because it is a more modifiable factor. In data from the Medicare Current Beneficiary Survey, CRN was reported by $11.5 \%$ of participants. Non-adherence has been associated with decreased quality of life, increased costs, and increased mortality $[11,12]$.

\section{Factors Contributing to Medication Non- Adherence and Strategies to Improve Adherence}

Non-adherence $[8,13]$ may be related to problems with the healthcare system including a poor quality of provider-patient relationships, poor communication, lack of access to healthcare and lack of continuity of care. Patient-physician interactions may be an important modifier of patient nonadherence. A survey of paired patient-physician respondents found that $61 \%$ of patients rarely or never 
discussed adherence with their physicians, and two-thirds of the patients had moderate or poor adherence. Although the physicians agreed that adherence was important, $67 \%$ were unaware of how often their patients missed medication [14]. Despite the potential role of the physician in promoting adherence, continuity of care with a physician was not associated with patient adherence to antihypertensive drugs among Medicare beneficiaries in a study of Part $D$ and Medicare claims data [15]. Patients who used multiple pharmacies were more likely to be non-adherent [16]. Factors that have been more consistently associated with non-adherence include female gender, Hispanic or nonWhite race, increased medication cost, increased dosing frequency, and using multiple different providers or pharmacies. Medication class has been a predictor as well, with some pharmacologic categories in a therapeutic class having less adherence due to side effects or other difficulties with administration (e.g., diuretics compared with other antihypertensive drugs). No consistent association with adherence has been shown for increasing age, and there is no association between the disease or disease risk level and adherence.

The medical condition is also contributory, with asymptomatic chronic disease and lack of physical clues associated with non-adherence, as well as mental health disorders such as depression. The patient categories relate to physical impairments such as vision problems or impaired dexterity or dysphasia; cognitive impairment; and psychological/behavioral issues. While increasing age is not a risk factor for non-adherence, older patients have age related issues that may pose unique barriers to adherence. For example, sensory losses, dysphagia, loss of cognitive function, and physical decline are all more common at elderly age and would provide unique barriers to optimal medication adherence [17]. Further, therapies not aligned with patient priorities for care provide added barriers to adherence and consideration must be given to deprescribing. The data cited above are likely most applicable to older adults who have a substantial life expectancy, few comorbid conditions, and who are generally physically fit and functional. An important consideration is that patients in this study were just older than age 50 and the extrapolation to a truly elderly population may be problematic. The recommended pharmacologic management in the cardiovascular clinical practice guidelines is diseasespecific, whereas the recommendations for an elderly patient with multiple comorbidities must be concordant with the healthcare priorities of that individual patient.

The therapy characteristics also contribute to non-adherence, particularly the complexity of the regimen and side effects. Socioeconomic/cultural contributions include low literacy, higher medication costs, poor social support, and attitudes/beliefs about medication [17].

Several strategies are available to increase adherence. These include reduction of medication cost, including the use of generic medications and lower co-pays and facilitating access to therapy such as better access to pharmacies, mail order, or assistance [18]. Simplification [19] of the dosing regimen such as once-daily dosing, convenient administration time, association with a clue such as meals, etc., are valuable strategies. Adherence aids, both low tech and high tech, include family help, pill boxes, calendars, alarms, and mobile applications. Of note, low-cost reminder aids have been shown to be less helpful than expected in improving adherence [20]. It is important to involve and educate the family and caregivers and to reinforce those therapies that are concordant with individual patient's goals of care.

Non-adherence in hypertension and cardiovascular disease is associated with adverse outcomes and increased healthcare costs. A study using claims data for patients with a recent myocardial infarction or known atherosclerotic disease demonstrated significant differences in major adverse cardiovascular 
events for up to 2 years based on being fully (C $80 \%$ ), partially (C 40 to $\backslash 80 \%$ ), or non-adherent ( $\backslash 40 \%$ ) to statins or angiotensin-converting enzyme inhibitors [21]. The association between non-adherence and outcome has varied by the measure used; one study showed that claims-based adherence measures of antihypertensive adherence were associated with cardiovascular events, but self-reported adherence was not [22]. A recent meta-analysis confirms the association between better adherence to b-blockers, angiotensin-converting enzyme inhibitors or angiotensin receptor blockers, antiplatelet drugs, and statins is associated with a lower risk of all-cause mortality, lower risk of cardiovascular mortality, lower risk of myocardial infarctions, and lower risk of hospitalization, using data pooled from ten studies including 106,002 patients with cardiovascular disease [23]. These data may be highly relevant for provider-elderly patient discussions; whereas mortality issues may be deemed less appropriate by some elderly patients, the lower risks of hospitalization are likely valued by most.

\section{Approaches to Optimizing Adherence in the Elderly Cardiovascular Disease Population}

Adherence is the extent to which patients follow recommendations or prescriptions. Adherence can be measured multiple ways and can be measured relatively easily, quickly, and in real-time clinical practice. Many studies of non-adherence use administrative claims or refill data to generate a numerical estimate of adherence. Most studies report the proportion of days covered (PDC), which is calculated with the days' supply obtained by the patient in a specific period as the numerator and the number of days in the specific period as the denominator. Another method is the Medication Possession Ratio, which is a sum of the days' supply obtained as the numerator divided by the number of days between the first and last prescription fill dates as the denominator. These methods capture different aspects of adherence than self-report, medication diaries, or pill-counting techniques, but there is reasonable correlation among the measures [24].

While for the most part older adults do well with adherence to medications, they are prescribed considerably more medications than younger patients. This more intense pharmacotherapy in older adults is largely due to the greater numbers of chronic diseases, especially cardiovascular disorders. Rigorously tested interventions are needed to assist elderly persons with medication adherence.

Marcum et al. recently conducted an evidence-based review of randomized controlled trials of medication adherence and associated health outcomes in older adults (mean age of the study groups was C 65 years) from 1970 through June 2016 [25]. Included trials were those with medication adherence as a primary or secondary variable and a health outcome such as exacerbation of disease, health-related quality of life, disability, an economic endpoint, or death. Of the 3305 potentially eligible studies reviewed, only 12 fulfilled inclusion criteria (0.36\%) and of these studies only three assessed cardiovascular disorders such as hypertension or heart failure. However, six of the 12 studies targeted adherence related to polypharmacy and it might be argued that many of those older adult patients had cardiovascular disorders. The interventions were classified into three groupings, namely behavioral or educational approaches $(n=3)$, pharmacist support $(n=7)$, and use of reminders or simplification strategies $(n=2)$. A key conclusion from this review is that there is a paucity of high-quality research (e.g., randomized controlled trials) that examined the association between medication adherence and relevant health outcomes in older adults, particularly those with cardiovascular disease.

Among the more effective interventions within the Marcum review was a study in patients with heart failure by Murray et al. [26]. The Murray study involved 314 low-income patients $\geq 50$ years of age who were followed for 1 year after randomization to usual care or intervention. 
The intervention had three basic components: patient education, therapeutic monitoring, and communication with providers. Special icon-based medication instruction materials stressed content, language, organization, and presentation of the medication information to participants in the intervention group. An icon or glyph such as the Ace of Hearts was placed on the ace-inhibitor medication container and that same icon inserted on the instructional materials provided to participants. Other cardiovascular medications targeted (and given unique icons) included angiotensinreceptor blockers, b-blockers, calcium-channel blockers, diuretics, and spironolactone. Adherence was measured by electronic monitors on the prescription containers, self-report, and refill adherence. Health outcomes measured included health-related quality of life, exacerbation of heart failure requiring an emergency department visit or hospitalization, patient satisfaction, ADEs, and annual total direct costs. Compared with usual care, medication adherence improved in the intervention group, which was associated with reduced composite emergency department visits and hospitalization resulting in lower direct healthcare costs. Patient satisfaction also improved. There were trends favoring reductions in ADEs and improved health-related quality of life that were not statistically significant. Because the Murray study involved patients older than 50 years of age, it remains uncertain how much of these data can be extrapolated to a truly elderly population.

There are few randomized controlled studies of medication adherence and associated health outcomes involving older adults with cardiovascular disease. Most of the effective studies in the review by Marcum et al. [25] involved behavioral/educational or pharmacist involved interventions such as the one conducted by Murray et al. [26]. The interventions were designed with attention to cognitive behavioral approaches and often involved tailoring to individual patient needs. Such interventions take considerable time for already overwhelmed health professionals and there is limited funding earmarked to subsidize their needed involvement. Automation will likely play an important future role in assisting patients with their medications; however, there are limited automation and informatics- driven solutions at this time. Many factors are at the root of poor medication adherence in the elderly such as inability of patients to manage multiple medications, polypharmacy often involving multiple prescribers, the cost of drugs, the lack of attention to health literacy, and the limited amount of time available to health professionals to address medication adherence problems in their patients.

Future rigorously designed studies of medication adherence and health outcomes should consider these factors in the design of adherence interventions, including the effects of deprescribing.

Adherence in cardiovascular disease is critical for disease maintenance and prevention of adverse outcomes, including emergency visits, hospitalizations, and mortality [10]. Recent data from the Medicare Part D program evaluating adherence in patients aged 65 years and older show a $26 \%$ nonadherence rate to antihypertensive medications, with substantial variation by state and by racial/ ethnic groups. Adherence varied by class of medication, with higher non-adherence for diuretics than other antihypertensives [27]. A wealth of literature has supported the importance of adherence in patients with hypertension and with coronary artery disease. The optimal threshold for good adherence is not established, but many studies identify adherence rates of $80 \%$ or higher as being adherent, or fully adherent (as opposed to non-adherence or partially adherence for rates $<80 \%$ ).

\section{Medication Management in the Older Adult with Cardiovascular Disease}


Cardiovascular medicine has been at the forefront of advancing the scientific underpinnings of clinical medicine. Principally through the conduct of randomized clinical trials that have been shown to reduce morbidity and enhance survival in patients with cardiovascular disease, there are a plethora of therapies to potentially offer patients with cardiovascular disease. Such successes have led to medication regimens in the rapidly expanding population of older adults with cardiovascular disease, the majority of who (68\%) also have multiple chronic conditions, that are increasingly long and complicated.

Polypharmacy, as evidenced by the case study presented, is increasingly common and presents significant challenges to the optimal management of drug therapies in older adults with cardiovascular disease. Indeed, a recent analysis of Veteran Affairs (VA) patients over the age of 65 years in 2007 showed that half of patients $(50 \%)$ had one or more prescribing problems including potentially inappropriate medications for older adults based on Beer's criteria, drug dosing, drug-drug interactions, drug-disease interaction, and high-risk drugs (warfarin, insulin, digoxin). Notably, chronologic age was not strongly associated with four of the five types of prescribing issues assessed and comorbid burden substantially increased risk only for drug-disease interactions and use of high-risk drugs. However, the number of drugs was consistently the strongest predictor of prescribing problems [28]; those patients in the highest quartile of medication use had a 6.6- to 12.5-fold greater risk of each type of prescribing problem compared with patients in the lowest quartile [17]. These data coupled with the recent report that demonstrates significant gaps in the guidelines for common cardiovascular disorders [29], which notes individuals aged 75 years and older have been markedly underrepresented in most major cardiovascular trials, and virtually all trials have excluded older adults with complex comorbidities and significant physical or cognitive disabilities. Accordingly, we have a limited database from which to draw upon in making critical decisions on drug therapy for the older adult and further research in this arena is desperately needed.

\section{Decision-Making in the Care of Older Adults with Multiple Chronic Conditions: Role of Patient Goals of Care in Shared Decision-Making}

Disease-specific and evidence-based guidelines are generally applicable to older adults with life expectancy $>10$-years, few comorbid conditions, and who are fit and functional, as long as they are consistent with patient preferences. At the other end of the spectrum, de-escalation of treatment, palliative care, and symptom management are appropriate for those with \1-2 years of life expectancy and advanced/end-stage disease such as dementia, cancer, or heart failure.

The uncertainty relates to those individuals who have a shorter than a decade life expectancy, an increasing number and severity of associated conditions, and an impairment of function, where patient priority aligned care is critical for the planning of pharmacotherapy [30]. It is in this population where deprescribing becomes an important component of care.

Factors for consideration in deprescribing include the drug burden, with consideration of nonpharmacologic therapies; symptoms suggesting adverse drug effects; high-risk drugs or combinations; ascertaining whether the benefits outweigh the harm; issues of the current benefit for disease and symptoms-is there unnecessary continuation as a consequence of a prescribing cascade [31]. Preventive therapies must be queried in the setting of limited life expectancy, with consideration of the time to benefit [32]. As well, it is important to identify whether the patient is actually taking the drug and the correct dosage of the drugs. 
Some patient goals of care may be to decrease symptoms and hospitalizations [33]; other patients may desire an increase in functional capacity and independence. Most value the maintenance of cognition and quality of life, whereas some value an improvement in survival. The importance is to individualize.

\section{Conclusions}

Cardiovascular disease increases incrementally with age. Older adults with cardiovascular disease who characteristically take multiple medications for multimorbidity face challenges of adherence and ADEs. Guideline-based medical management for cardiovascular disease is disease-specific; this differs markedly for elderly patients with multimorbidities where patient goals of care must align with medication management. Particularly for elderly individuals with limited life expectancy, a deprescribing approach, in consultation with the patient, is likely to ensure patient-preferred care. 


\section{References}

1. Benjamin EJ, Blaha MJ, Chiuve SE, et al. Heart disease and stroke statistics-2017 update: a report from the American Heart Association. Circulation. 2017;135:e146-605.

2. Nahin RL, Pecha $M$, Welmerink DB, et al. Concomitant use of prescription drugs and dietary supplements in ambulatory elderly patients. JAGS. 2009;57:1197-205.

3. Rochon PA, Gerwitz JH. The prescribing cascade revisited. Lancet. 2017;389:1778-80.

4. Qato DM, Wilder J, Schumm LP, et al. Changes in prescription and over-the counter medication and dietary supplement use among older adults in the United States, 2005 vs 2011. JAMA Intern Med. 2016;176:473-82.

5. Budnitz DS, Lovegrove MC, Shehab N, et al. Emergency hospitalizations for adverse drug events in older Americans. N Engl J Med. 2011;365:2002-12.

6. Shehab N, Lovegrove MC, Geller Al, et al. US Emergency Department visits for outpatient adverse drug events, 2013-2014. JAMA. 2016;316:2115-25.

7. Aitken M, Valkova S. Avoidable costs in US Healthcare: the $\$ 200$ billion opportunity from using medicines more responsibly. Report by the IMS Institute for Healthcare Informatics, June 2013. http://www.imshealth.com/files/web/IMSH\%20Institute/Reports/Avoidable_Costs_in\%20_US_Healthca re/IHII_AvoidableCosts_2013.pdf. Accessed 15 May 2017.

8. Sabate E. Adherence to long-term therapies: evidence for action. Geneva: World Health Organization; 2003.

9. Tamblyn R, Eguale $T$, Huang A, et al. The incidence and determinants of primary nonadherence with prescribed medication in primary care: a cohort study. Ann Intern Med. 2014;160:441-50.

10. Ho PM, Bryson CL, Rumsfeld JS. Medication adherence: its importance in cardiovascular outcomes. Circulation. 2009;119:3028-35.

11. Marcum ZA, Zheng Y, Perera S, For the Health ABC Study, et al. Prevalence and correlates of selfreported medication non-adherence among older adults with coronary heart disease, diabetes mellitus, and/or hypertension. Res Social Adm Pharm. 2013;9:817-27.

12. Madden JM, Graves AJ, Zhang F, et al. Cost-related medication nonadherence and spending on basic needs following implementation of Medicare Part D. JAMA. 2008;299:1922-8.

13. Hughes S. Compliance with drug treatment in the elderly. Prescriber. 1998;9:45-54.

14. Hines R, Stone NJ. Patients and physicians beliefs and practices regarding adherence to cardiovascular medication. JAMA Cardiol. 2016;1:470-3.

15. Robles S, Anderson GF. Continuity of care and its effect on prescription drug use among Medicare beneficiaries with hypertension. Med Care. 2011;49:516-21.

16. Marcum ZA, Driessen J, Thorpe CT, et al. Effect of multiple pharmacy use on medication adherence and drug-drug interactions in older adults with Medicare Part D. J Am Geriatr Soc. 2014;62:244-52. 
17. Steinman MA, Hanlon JT. Managing medications in clinically complex elders: "There's got to be a happy medium". JAMA. 2010;304:1592-601.

18. Choudhry NK, Avorn J, Glynn RJ, et al. Full coverage for preventive medications after myocardial infarction, for the Post- Myocardial Infarction Free Rx Event and Economic Evaluation (MI FREEE) Trial. N Engl J Med. 2011;365:2088-97.

19. Wolf MS, Curtis LM, Waite K, et al. Helping patients simplify and safely use complex prescription regimens. Arch Intern Med. 2011;171:300-5.

20. Choudhry NK, Krumme AA, Ercole PM, et al. Effect of reminder devices on medication adherence: The REMIND randomized clinical trial. JAMA Intern Med. 2017;177:624-31.

21. Bansilal S, Castellano JM, Garrido E, et al. Assessing the impact of medication adherence on longterm cardiovascular outcomes. J Am Coll Cardiol. 2016;68:789-801.

22. Krousel-Wood M, Holt E, Joyce $\mathrm{C}$, et al. Differences in cardiovascular disease risk when antihypertensive medication adherence is assessed by pharmacy fill versus self-report: the Cohort Study of Medication Adherence among Older Adults (CoSMO). J Hypertens. 2015;33:412-20.

23. Du L, Cheng Z, Zhang Y, et al. The impact of medication adherence on clinical outcomes of coronary artery disease: A meta-analysis. Eur J Prev Cardiol. 2017;24:962-70.

24. Marcum ZA, Sevick MA, Handler SM. Medication nonadherence: a diagnosable and treatable medical condition. JAMA. 2013;309:2105-6.

25. Marcum ZA, Hanlon JT, Murray MD. Improving medication adherence and health outcomes in older adults: an evidence-based review of randomized controlled trials. Drug Aging. 2017;34:191-201.

26. Murray MD, Young J, Hoke $S$, et al. Pharmacist intervention to improve medication adherence in heart failure: a randomized trial. Ann Intern Med. 2007;146:714-25.

27. Ritchey $M$, Chang A, Powers $C$, et al. Vital signs: disparities in antihypertensive medication nonadherence among medicare part $d$ beneficiaries-United States, 2014. MMWR Morb Mortal Wkly Rep. 2016;65:967-76.

28. Scott IA, Hilmer SN, Reeve E, et al. Reducing inappropriate polypharmacy: the process of deprescribing. JAMA Intern Med. 2015;175:827-34.

29. Rich MW, Chyun DA, Skolnick AH, et al. Knowledge gaps in cardiovascular care of older adults: a Scientific Statement from the American Heart Association, American College of Cardiology, and American Geriatrics Society: executive summary. JAGS. 2016;64:2185-92.

30. Tinetti ME, Bogardus ST Jr, Agostini JV. Potential pitfalls of disease-specific guidelines for patients with multiple conditions. N Engl J Med. 2004;351:2870-4.

31. Rochon PA, Gurwitz JH. Optimizing drug treatment for elderly people: the prescribing cascade. BMJ. 1997;315:1096-9.

32. Holmes HM, Min LC, Yee M, et al. Rationalizing prescribing for older patients with multimorbidity: considering time to benefit. Drug Aging. 2013;30:655-66. 
33. Lipman HI, Kalra A, Kirkpatrick JN. Foundations of medical decision-making for older adults with cardiovascular disease. J Geriatr Cardiol. 2015;12:335-9. 
Table 1. Case of an elderly woman with a complicated medical regimen

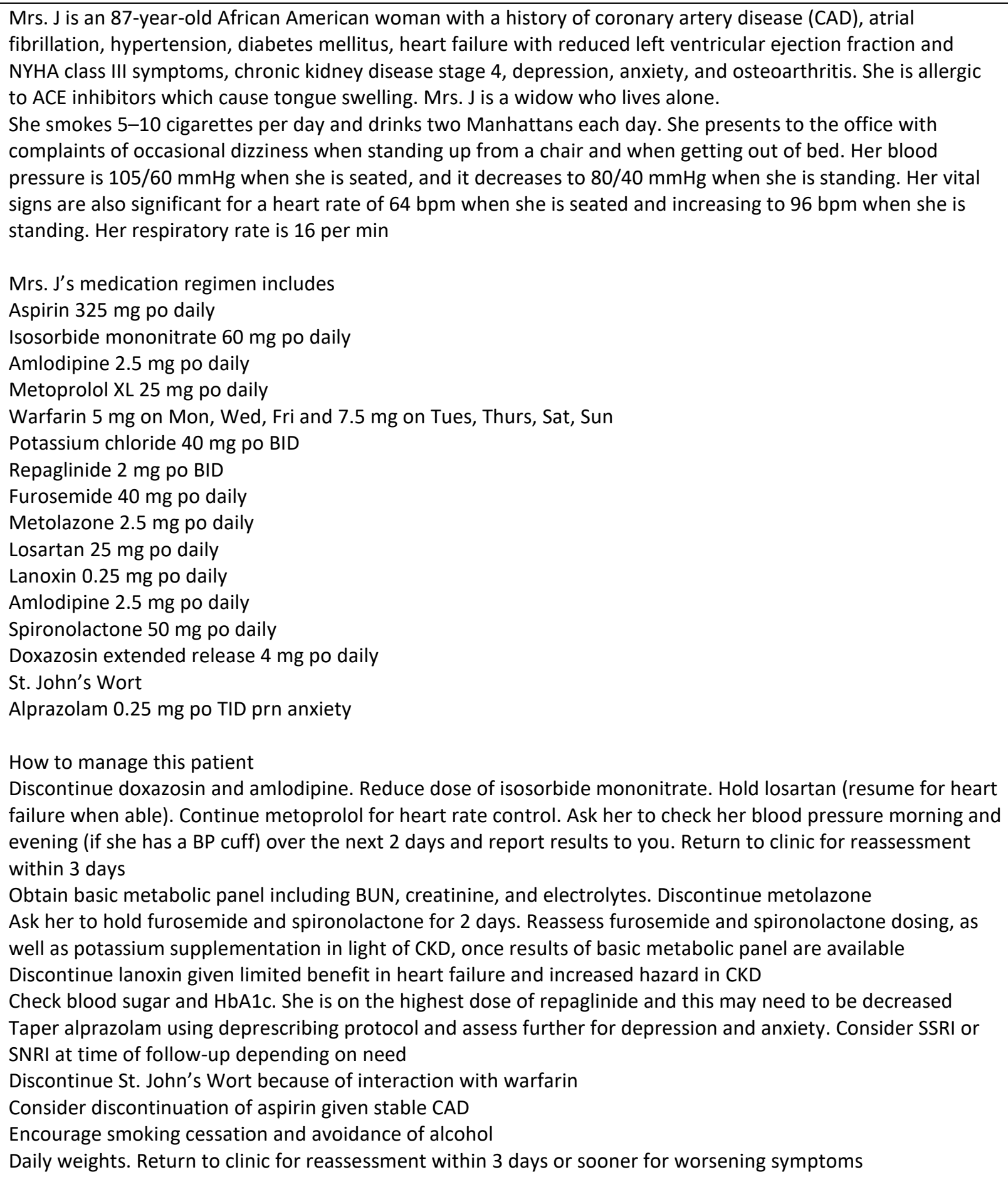

$A C E$ angiotensin-converting enzyme, $B I D$ twice daily, $B P$ blood pressure, $b p m$ beats per minute, $B U N$ blood urea nitrogen, $C A D$ coronary artery disease, $C K D$ chronic kidney disease, NYHA New York Heart Association, po orally, prn when necessary, SNRI serotonin-norepinephrine reuptake inhibitor, SSRI selective serotonin reuptake inhibitor, TID three times daily 
Table 2. Physiologic change: adverse drug effects

\begin{tabular}{lll}
\hline Aging physiologic change & Drug & ADEs \\
\hline Conduction system degeneration & CCBs and $\beta$-blockers & Bradycardia \\
Arterial and ventricular stiffness & NSAIDs & Heart failure and fluid retention \\
Baroreflex and balance & Antihypertensives & Falls and orthostatic hypotension \\
Decreased thirst & Diuretics & Dehydration \\
Gastric atrophy & Anticoagulation & Gl bleeding \\
\hline ADEs adverse drug effects, CCBs calcium channel blockers, Gl gastrointestinal, NSAIDs non-steroidal anti-inflammatory drugs
\end{tabular}

Table 3. Drug-disease adverse events. Drugs relatively contraindicated in selected diseases

\begin{tabular}{ll}
\hline Drug & Disease \\
\hline$\beta$-blockers & Asthma, COPD \\
ACE-I, NSAIDs & Renal insufficiency \\
Glucocorticoids & Diabetes \\
Anticholinergics & BPH, cognitive impairment \\
CCBs & Constipation \\
& Gastroesophageal reflux \\
Warfarin & Low albumin states (malnutrition) \\
\hline
\end{tabular}

$A C E-I$ angiotensin-converting enzyme inhibitor, $B P H$ benign prostatic hyperplasia, $C C B s$ calcium channel blockers, $C O P D$ chronic obstructive pulmonary disease, NSAIDs non-steroidal anti-inflammatory drugs

Table 4. Drug-nutraceutical/ OTC interactions

\begin{tabular}{|c|c|c|}
\hline Herbal & Drug & Effect \\
\hline \multirow[t]{4}{*}{ St. John's Wort } & Warfarin & 个Bleeding \\
\hline & $\mathrm{CCB}$ & $\downarrow$ Absorption/个 metabolism \\
\hline & Statins & $\downarrow$ Levels (CYP3A4) \\
\hline & Amiodarone & Photosensitivity \\
\hline \multirow[t]{3}{*}{ Gingko Biloba } & Warfarin & 个Bleeding \\
\hline & $\mathrm{CCB}$ & 个Absorption \\
\hline & Statins & 个Levels (CYP3A4) \\
\hline \multirow[t]{2}{*}{ Garlic } & HMG-CoA reductase inhibitors & 个Levels (CYP3A4) \\
\hline & Aspirin, warfarin & 个Bleeding \\
\hline \multirow[t]{3}{*}{ Ginseng } & $\mathrm{CCB}$ & 个Absorption/ $\downarrow$ metabolism \\
\hline & Digoxin & 个Levels \\
\hline & Warfarin & $\downarrow$ INR \\
\hline Vitamin E & Warfarin & Bleeding \\
\hline \multirow[t]{3}{*}{ NSAIDs } & Warfarin, aspirin & Bleeding \\
\hline & ACE-I & Renal failure \\
\hline & Steroids & $\downarrow \mathrm{Na}$, volume retention \\
\hline Psyllium & Aspirin & $\downarrow$ Absorption \\
\hline
\end{tabular}

ACE-I angiotensin-converting enzyme inhibitor, $C C B$ calcium channel blocker, HMG-COA 3-hydroxy-3-methylglutaryl coenzyme A, INR international normalized ratio, NSAIDs non-steroidal anti-inflammatory drugs, OTC over the counter products, $\uparrow$ increase, $\downarrow$ decrease 